\title{
Myocarditis in the Presence of Respiratory Syncytial Virus (RSV) Infection: A Case Study
}

\author{
Sean O’Brien ${ }^{1}$, Samer Ajam², Amy Han ${ }^{3}$ \\ ${ }^{1}$ Indiana University School of Medicine; ${ }^{2}$ Community Care Network, Community Health Partners; \\ ${ }^{3}$ Indiana University School of Medicine, Department of Psychiatry
}

\section{Background/Objective:}

Respiratory syncytial virus (RSV) causes acute respiratory infections in children and adults. RSV has many non-specific symptoms such as cough and dyspnea. RSV is associated with high mortality in children, the elderly, and immunocompromised individuals. Although rare, RSV has been reported to cause extrapulmonary complications such as arrhythmias and myocarditis. This case focuses on a patient infected with RSV who presents with acute sustained monomorphic ventricular tachycardia (SMVT).

\section{Case Overview:}

An 83 year-old patient with a history of type 2 diabetes mellitus, hypertension, persistent atrial fibrillation, and asthma presented to the emergency department with concerns of cough, malaise, and a syncopal episode. Upon admission, the patient tested positive for RSV and was diagnosed with acute bronchitis exacerbated by history of asthma. The patient soon developed SMVT with heart rates as high as 235 beats per minute. After consultation with an electrophysiologist, the VT was attributed to myocarditis as a result of the RSV infection. Oral amiodarone was prescribed, and the patient was discharged two weeks later. In a follow-up visit, no SMVT was reported, and the dosage of amiodarone was decreased.

\section{Discussion:}

With the onset of the SARS-CoV2 pandemic in early 2020, myocarditis associated with viral infection has been of interest in recent literature. Many cases of cardiovascular complications have been reported in patients infected with SARS-CoV2. Consequently, it is important to discuss cases of other respiratory viruses also presenting with arrhythmia and myocarditis. In the current case, a patient with RSV developed new onset VT. VT can be life-threatening and can cause further cardiovascular complications.

\section{Conclusion:}

RSV can cause new onset cardiovascular complications, albeit rare. It is important for clinicians to be aware of such complications especially in cases in which patients have preexisting cardiovascular conditions. Patients infected with RSV should be closely monitored for new onset complications. 